Received: $\quad 2019.08 .25$ Accepted: 2019.10.10 Published: 2019.11.01

\title{
Overcoming Hepatic Artery Thrombosis After Living Donor Liver Transplantations: An Experience from Asan Medical Center
}

Division of Hepatobiliary Surgery and Liver Transplantation, Department of Surgery, Asan Medical Center, University of Ulsan College of Medicine, Seoul, South Korea
Corresponding Author: Source of support:

$\begin{aligned} \text { ABCDEF } & \text { Gil-Chun Park } \\ \text { ABCDEF } & \text { Deok-Bog Moon } \\ \text { ABCD } & \text { Sang-Hyun Kang } \\ \text { BCD } & \text { Chul-Soo Ahn } \\ \text { BCD } & \text { Shin Hwang } \\ B C D & \text { Ki-Hun Kim } \\ B C D & \text { Tae-Yong Ha } \\ B C D & \text { Gi-Won Song } \\ B C D & \text { Dong-Hwan Jung } \\ \text { ABCD } & \text { Yong-In Yoon } \\ A B C D & \text { Sung-Gyu Lee }\end{aligned}$

ABCDEF Gil-Chun Park

Study Design A Data Collection B Statistical Analysis C Data Interpretation D Manuscript Preparation E Literature Search F Funds Collection G

Background: Hepatic artery (HA) reconstruction in living donor liver transplantation (LDLT) is more technically demanding than deceased donor LT (DDLT) because of the small diameter and short HA stump of the partial liver graft. Hence, hepatic artery thrombosis (HAT) can occur infrequently even though the HA is reconstructed microscopically. HAT is closely related to graft failure and mortality. Therefore, HAT should be detected early and HA flow reconstituted using several arterial inflows. We successfully performed redo HA reconstruction in LDLT and report our management process and outcomes.

Material/Methods: The right gastroepiploic artery (RGEA) was used in 15 patients, previous native HA in 3, and interposition graft from the aorta in 1. All HA reconstructions were performed under a microscope using the end-to-end interrupted suture method. We reviewed technical feasibility, cause of hepatic artery revision (HAR), patency of redo HA flow, graft salvage rate, time of revision, biliary complications, and mortality.

Results: Ten of 21 cases were salvage LT. Biliary complications developed in 6 cases. The mean interval of HAR with the RGEA was $1.5 \pm 1.2$ postoperative days. All patients were alive without lethal complications of HAT during the mean follow-up period of 23.3 months. In the other 6 cases of HAR without using the RGEA, we performed redo HA reconstruction after thrombectomy with the native right $\mathrm{HA}(\mathrm{n}=2)$, right gastric artery, left HA, gastroduodenal artery, and jump graft from the aorta ( $n=1$, respectively). Among them, 3 died from biliary sepsis, graft dysfunction from large-sized ischemic injury, and pneumonia.

Conclusions: HAR with the RGEA is feasible for HAT management in LDLT patients without adequate hepatic arteries. When all inflows mentioned are unavailable, jump graft from the aorta using a cadaveric fresh iliac artery may be feasible.

MeSH Keywords: $\quad$ Gastroepiploic Artery • Hepatic Artery • Liver Transplantation • Living Donors • Thrombosis

Full-text PDF: https://www.annalsoftransplantation.com/abstract/index/idArt/919650

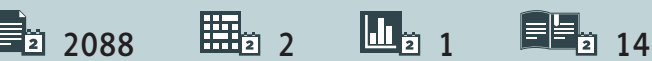




\section{Background}

Because of the shortage of deceased organ donation in Asian countries, living donor liver transplantation (LDLT) is mostly performed; for successful outcomes, we should ensure sufficient arterial inflow and perform hepatic artery $(\mathrm{HA})$ reconstruction because hepatic arterial complications affect graft and patient survival. Hepatic artery thrombosis (HAT) after LDLT is a lethal complication, with incidence ranging from $2.5 \%$ to $9 \%[1,2]$. Liver abscess or bile duct (BD) strictures, as well as graft necrosis and failure, can be related to HAT as clinical manifestations [3]. Although we attempt to meticulously dissect the HA without inflicting any arterial injury, the native healthy $\mathrm{HA}$ is sometimes unavailable because of repeated transarterial chemoembolization (TACE), radiation therapy, or other factors causing intimal injury to the recipient's HA. In this difficult situation, for obtaining the hepatic arterial source, instead of the native $\mathrm{HA}$, the right gastroepiploic artery (RGEA) is mostly preferred because of its ease of access and adequate length [4]. In our institution, the RGEA has been used to replace or supplement arterial sources for LDLT in cases of multiple-graft arteries and insufficient hepatic arterial flow of the recipient, as well as hepatic artery revision (HAR) for HAT. Therefore, we retrospectively analyzed outcomes of HAR using the RGEA and evaluated the proper surgical management of hepatic artery occlusion in patients after LDLT.

\section{Material and Methods}

We retrospectively analyzed a total of 1232 cases of adult LT from January 2016 to June 2019, including 1101 right-lobe grafts cases, 26 left-lobe grafts cases, 104 dual cases, and 1 wholeliver graft case. Among 1232 cases, 21 cases of HAR were reviewed for clinical characteristics and outcomes. At our institution, intraoperative Doppler ultrasonography is performed after arterial reconstruction, and daily Doppler ultrasonography until postoperative 5 days and dynamic computed tomography $(\mathrm{dCT})$ with three-dimensional vascular reconstruction at least once per week after LT are routinely performed as imaging study protocol. If hepatic arterial occlusion is suspicious on Doppler ultrasonography following LDLT, we evaluate hepatic artery inflow and confirm HAT using dCT and celiac angiography while waiting for surgical revascularization or during an additional evaluation, when required. In our center, surgical revascularization is, in principle, preferable to radiological HA intervention for HAT management because it can cause iatrogenic HA injuries and there is no definite treatment. When we performed HAR for HAT, the native hepatic artery after thrombectomy was considered as the first option for hepatic inflow if HAT itself was a cause of HAR and not HA dissection, as detected in the pre-HAR studies. HAT was localized into the right or left HA during HAR. The RGEA was regarded as the second option, and a graft from the aorta was used as the last arterial alternative, if available. Transplant surgeons performed all arterial reconstructions under a microscope. During initial LT, HA reconstruction methods, including preparing donor and recipient hepatic arterial stumps, were followed as stated in a previous study [5]. After splenic arterial embolization or ligation, which was basically performed in the presence of poor HA flow or HAR for the augmentation of the hepatic artery or RGEA blood flow, the RGEA was dissected from the greater curvature of the stomach and greater omentum with its full length after selecting the dissection initiating site by manual palpation and direct visualization of the proper size along the RGEA running course. After dissection of the anastomotic site of the RGEA, a clamp was applied in the distal portion for $>10 \mathrm{~min}$ for natural enlargement of the diameter. We usually used nylon $9-0$ or $10-0$ sutures following an end-to-end technique [4]. Size mismatches between the 2 arterial ends were overcome by direct dilation of the RGEA stump or using a branch-patch from the gastric omental branch of the RGEA. During non-rotation of the graft arterial clamp because of graft HA stump shortening after the previous anastomosis was resected from the proximal and distal parts, a back-wall support suture technique was applied [6]. A continuous suture technique was not indicated. During HAR for HAT, the breakup of the existing BD anastomosis and redo $B D$ reconstruction was necessary so that enough HA stump length was available for HAR with native HA after thrombectomy, and proper position or direction of the HA inflow without angulation was required. However, redo $B D$ reconstruction was mostly unnecessary in HAR with the RGEA. While using the aorta jump graft for the HA inflow, after the anterior wall of the infrarenal aorta near the Treitz ligament was dissected with proximal and distal flow control, a continuous suture was applied with Prolene 6-0 at the proximal inflow between the fresh iliac artery from the cadaver and aorta. The iliac conduit was positioned posterior to the transverse colon and stomach through the mesocolon. HAR was performed between the iliac conduit using matching-sized branch and graft HA. After redo HA reconstruction, follow-up imaging studies were performed as per the routine protocol. Low-molecular-weight heparin (enoxaparin sodium, $40 \mathrm{mg} \mathrm{SC} \mathrm{q} 24 \mathrm{hr}$ ) and prostaglandin E1 (alprostadil, $32 \mathrm{mcg} / \mathrm{hr}$ ) were administered to all patients. The study was approved by the Institutional Review Board of the Asan Medical Center (S2019-1713-0001).

\section{Results}

Among the overall 1431 arterial anastomoses, including 105 cases of dual grafts and 1 whole-liver graft, we experienced $21(1.4 \%)$ cases of HAR due to hepatic artery occlusion after adult LTs. Out of the 21 cases of HAR, the RGEA was used in 15 patients. Eleven patients were men and 4 were women. 
Table 1. Characteristics of the 21 patients who underwent HAR with RGEA and other inflows.

\begin{tabular}{|c|c|c|c|}
\hline Characteristics & RGEA group ( $n=15)$ & Non-RGEA group $(n=6)$ & Comment \\
\hline \multicolumn{4}{|l|}{ Sex } \\
\hline Male/Female & $11 / 4(73.3 \% / 26.7 \%)$ & $5 / 1(83.3 \% / 16.7 \%)$ & \\
\hline Age, years & $58.7 \pm 7.1$ & $58.0 \pm 7.8$ & \\
\hline \multicolumn{4}{|l|}{ Diagnosis } \\
\hline Viral hepatitis & 9 (60.0\%) & $5(83.3 \%)$ & \\
\hline Alcoholic & $3(20.0 \%)$ & 0 & \\
\hline Others & $3(20.0 \%)$ & $1(16.7 \%)$ & \\
\hline MELD score & $10.0 \pm 3.4$ & $14.8 \pm 10.7$ & \\
\hline GRWR, \% & $0.94 \pm 0.17$ & $1.14 \pm 0.50$ & \\
\hline Mean follow up, mo & 23.3 (range, $1-41$ ) & 9.5 (range, 3-18) & \\
\hline \multicolumn{4}{|l|}{$\mathrm{HCC}$} \\
\hline Yes/no & $10(66.7 \%) / 5(33.3 \%)$ & $3(50 \%) / 3(50 \%)$ & \\
\hline \multicolumn{4}{|l|}{ preLT therapy } \\
\hline TACE/RFA/Radiation & $10(66.7 \%) / 2(13.3 \%) / 1(6.7 \%)$ & $3((50 \%) / 0 / 0$ & \\
\hline \multicolumn{4}{|l|}{ Graft type } \\
\hline $\mathrm{mRL} / \mathrm{LL} /$ dual/whole & $13(86.7 \%) / 1(6.7 \%) / 1(6.7 \%) / 0$ & $4(66.7 \%) / 1(16.7 \%) / 0 / 1(16.7 \%)$ & \\
\hline \multicolumn{4}{|l|}{ Salvage } \\
\hline Yes/no & $5(33.3 \%) / 10(66.7 \%)$ & $4(66.7 \%) / 2(33.3 \%)$ & \\
\hline Total Ischemic time, min & $133.2 \pm 33.4$ & $169.0 \pm 104.4$ & nonRGEA: 1 whole graft \\
\hline \multicolumn{4}{|l|}{ Cause of HAR } \\
\hline HAT & $10(66.7 \%)$ & $4(66.7 \%)$ & \\
\hline HAD & $4(26.7 \%)$ & $1(16.7 \%)$ & \\
\hline Kinking/compression & $1(6.7 \%) / 0$ & $0 / 1(16.7 \%)$ & \\
\hline Interval, day & $1.5 \pm 1.2$ & $1.1 \pm 0.8$ & \\
\hline \multicolumn{2}{|l|}{ Method } & & RGEA group only \\
\hline \multicolumn{4}{|l|}{ Number of bile duct } \\
\hline Single/double & $11(73.3 \%) / 4(26.7 \%)$ & $5(83.3 \%) / 1(16.7 \%)$ & \\
\hline \multicolumn{4}{|l|}{ Type of anastomosis } \\
\hline Duct to duct & $12 \quad(80 \%)$ & $3 \quad(50 \%)$ & \\
\hline Hepaticojejunostomy & $3 \quad(20 \%)$ & $3 \quad(50 \%)$ & \\
\hline \multicolumn{4}{|l|}{ Biliary complications } \\
\hline Stricture & $5(33.3 \%)$ & 0 & \\
\hline Leak/biloma & 0 & $1(16.7 \%)$ & \\
\hline Hospital stay, day & $35.3 \pm 27.9$ & $21.1 \pm 12.9$ & \\
\hline 90-day patient survival & $100 \%$ & $50 \%$ & \\
\hline Mortality & $0 \quad(0 \%)$ & $3 \quad(50 \%)$ & $\begin{array}{c}\text { Biliary sepsis, graft } \\
\text { dysfunction, pneumonia }\end{array}$ \\
\hline
\end{tabular}

HAR - hepatic artery revision; RGEA - right gastroepiploic artery; MELD - model for end-stage liver disease; GRWR - graft-

recipient weight ratio; HCC - hepatocellular carcinoma; LT - liver transplantation; TACE - transcatheter arterial chemoembolization; RFA - radiofrequency ablation; $\mathrm{mRL}$ - modified right lobe; LL - left lobe; HAT - hepatic artery thrombosis; HAD - hepatic artery intimal dissection.

The mean age was 58.7 \pm 7.1 (range, $45-70$ ) years. Patients with hepatic artery occlusion undergoing HAR using the RGEA and other inflows are summarized in Table 1. The most common cause of HAR was HAT $(n=14)$, hepatic artery intimal injury including dissection at the recipient $(n=3)$, or graft $(n=2)$ side, and kinking from the redundancy of recipient's hepatic 
Table 2. Different studies on the morbidity and mortality from HAT after LDLT.

\begin{tabular}{|c|c|c|c|c|}
\hline & Incidence $(\mathrm{n}, \%)$ & Morbidity $(n, \%)$ & Mortality & Comment \\
\hline Song S, et al. [3] & $7 / 522(1.3 \%)$ & 3 BS (42.8\%) & $42.8 \%$ & \\
\hline Bekker J, et al. [7] & $4.4 \%$ & NS & $33.3 \%$ (range, 0-80\%) & Review article \\
\hline Uchiyama H, et al. [9] & $2 / 335(0.6 \%)$ & $\begin{array}{c}1 \text { BS (50\%) } \\
1 \text { re-LT (50\%) }\end{array}$ & No & \\
\hline Wang CC, et al. [13] & $3 / 126(2.4 \%)$ & $\begin{array}{c}1 \text { bile leak }(33.3 \%) \\
2 \text { BS }(66.6 \%)\end{array}$ & No & \\
\hline Lee JH, et al. [14] & $2 / 463(0.4 \%)$ & NS & $50 \%$ & \\
\hline Current study & $14 / 1,232(1.1 \%)$ & 5 BS (23.8\%) & $3 / 1,232(0.2 \%)$ & \\
\hline
\end{tabular}

BS - biliary stricture; NS - not stated; LT - liver transplantation.

artery and compressive occlusion after biliary reconstruction ( $n=1$, respectively). Among 15 cases of HAR using the RGEA, $10(66.7 \%)$ patients with hepatocellular carcinoma (HCC) as pre-LT management underwent repeated transcatheter arterial chemoembolization (TACE) preoperatively. The interval of HAR was $1.5 \pm 1.2$ postoperative (range, $0-5$ ) days. During HAR using the RGEA, antegastric reconstruction type was performed in $8(53.3 \%)$ patients and retrogastric type in 7 . There was no difference in the results for each type. All patients underwent interruption of the splenic arterial flow for redo HAR without splenic arterial modulation during initial LDLT. During the initial LDLT, 8 patients underwent splenic arterial interruption because of low GRWR, and hypersplenism.

In this study of 15 HAR cases using the RGEA, all patients were alive without any lethal complication or graft loss over a mean follow-up of 23.3 (range, 1-41) months. All reconstructed hepatic arteries using the RGEA showed good patency without re-thrombosis or stenosis. However, of 11 patients with a single BD opening in LDLT using mRL graft, biliary strictures occurred in 2 (18.1\%). In the other 6 cases of HAR without using the RGEA, we performed redo HA reconstruction after thrombectomy with the native right $\mathrm{HA}(n=2)$, right gastric artery, left $H A$, gastroduodenal artery, and jump graft from the aorta $(n=1$ each). Among those patients, a biliary complication occurred in $1(16.7 \%)$. Three patients died of biliary sepsis from an intrahepatic abscess and biloma caused by ischemic BD injury, pneumonia, and graft dysfunction from large-sized ischemic graft damage at the time of HAR while waiting for re-transplantation.

\section{Discussion}

In LDLT, while using partial liver graft, the graft HA is smaller and its stump is shorter than with a whole-liver graft. Therefore, extensive dissection of the recipient hepatic arteries is necessary for matching the size for arterial reconstruction.
This procedure may increase the risk of intimal dissection or transmural hematoma in the recipient $\mathrm{HA}$ in the situation of an enlarged and vulnerable HA caused by portal hypertension [4]. In addition, pretransplant TACE, radiofrequency ablation (RFA), or radiation therapy (RTX) for HCC management may incur hepatic arterial injuries. In this study, 13 (86.7\%) of 15 HAR patients underwent preoperative TACE or RFA, and RTx including salvage LDLT. However, an arterial injury may be avoided with delicate hilar dissection by transplant surgeons who are expert in microsurgery. In this study, hepatic artery dissection at the recipient side was a cause of HAR in only $3(14.3 \%)$ out of 21 cases, showing a relatively low incidence.

HAT is a fatal complication in LT, but the most efficient management of HAT is debated. Re-transplantation was the treatment of choice in early HAT. Only 1 case needed re-transplantation because of graft dysfunction from ischemic graft damage in our study. However, urgent re-arterialization of HAT should be considered as a first option to prevent re-transplantation when faced with a limited donor pool [3]. If early detection of HAT causes minimal graft ischemic injury, HAR may provide a good result. Several studies reported that mortality after diagnosis of HAT was $33.3 \%$ (range, $0-80 \%$ ) $[3,4,7,8]$. In our study, there was no mortality or re-transplantation in all patients who underwent urgent HAR using the RGEA for HAT. The excellent outcome resulted from HAR being performed in all cases within postoperative 5 days (mean interval, $1.5 \pm 1.2$ days). Early detection of HAT is essential to prevent graft or patient loss; therefore, we evaluated hepatic artery flow using daily Doppler ultrasonography up to postoperative 5 days and dCT at least once a week. When HAT was suspicious on Doppler, we immediately performed dynamic CT and hepatic angiography in consultation with radiologists. Before urgent HAR, splenic artery embolization for arterial flow and size augmentation was performed during celiac angiography if HAT was limited to the right or left HA and the RGEA was slender, as a policy for early HAT management. 


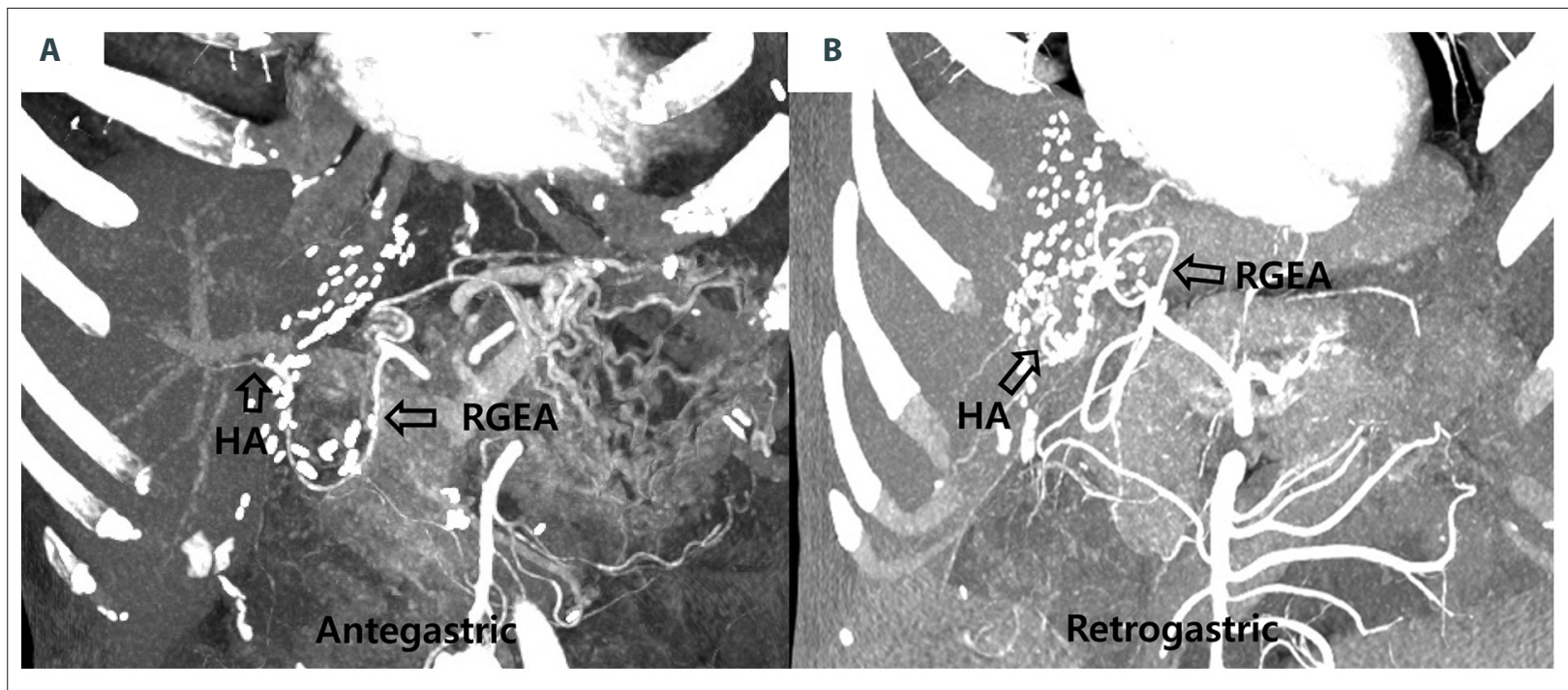

Figure 1. Follow-up dynamic computed tomography showing good patency of hepatic artery (HA) inflow after hepatic artery revision (HAR) using the right gastroepiploic artery (RGEA). According to the course of the RGEA, there are 2 methods for HAR: antegastric (A) and retrogastric (B).

The biliary stricture was noted in 5 (33.3\%) patients with HAR in the present study. However, of 11 patients with a single BD opening in LDLT using mRL graft, biliary strictures occurred in $2(18.1 \%)$. Our results were much better than those of another study showing biliary stricture in $50 \%$ of the patients [9] (Tables 1, 2), and those patients were successfully treated with endoscopic retrograde (ERBD) or percutaneous transhepatic (PTBD) biliary drainage.

As a substitute for the native hepatic arteries in recipients, RGEA, splenic artery, left gastric artery, middle colic artery, and various interposition grafts were suggested in various studies [10-12]. If HAT is not propagated down to proper HA, re-use of the recipient native HA is practicable. In our experience, other alternative $\mathrm{HA}$ inflows are not necessary if the native $\mathrm{HA}$ and the RGEA are used as HA inflow. When none of the HA inflows mentioned above are unavailable, a jump graft from the aorta using cadaveric fresh iliac artery may be a possible option. The RGEA may be a favorable option because it is easy to dissect without bleeding, and it has enough length to perform HAR [4]. An adequate length of the RGEA along with easy handling of the anastomosis direction can avoid the necessity of breakup of $B D$ reconstruction for a good operation field during HAR. We performed redo BD reconstruction in only 1 patient in our study, and that patient experienced biliary stricture and underwent ERBD and PTBD. We perform HAR using the RGEA using 2 methods, according to the running course of the RGEA: antegastric and retrogastric methods (Figure 1). Recently, the retrogastric method is preferred because it is similar to the natural running course of the recipient HA and has a low risk of anastomosis site kinking. Further, tension at the anastomosis site is less influenced by the distension of the stomach, duodenum, and large intestine. However, the antegastric type can be applicable in cases of severe adhesion to the hepatic hilum, similar to salvage LT.

Our study revealed good results of HAR using the RGEA for HAT compared to results of other studies, as shown in Table 2. The reasons for these good results were as follows: first, HAT was detected early using daily Doppler ultrasonography and dynamic CT or angiography in consultation with the radiologists. Second, urgent surgical revascularization was preferred to HA intervention by the radiologists. Urgent HAR can prevent graft dysfunction and patient loss. This is supported by the short interval time of HAR and less or no mortality (Table 1). Third, we selected proper arterial inflows for HAR according to the described indications for the $3 \mathrm{HA}$ inflows.

\section{Conclusions}

Urgent HAR using the 3 HA inflows (native HA, RGEA, and jump graft) for the treatment of hepatic artery occlusion is a feasible and successful procedure when there is an inadequate recipient hepatic artery during LDLT. The RGEA was found to be universally available for HA inflow, except in 1 patient.

\section{Conflicts of interest}

None. 


\section{References:}

1. Stange BJ, Glanemann M, Nuessler NC et al: Hepatic artery thrombosis after adult liver transplantation. Liver Transpl, 2003; 9(6): 612-20

2. Abou El-Ella K, Al Sebayel M, Ramirez C, Hessien R: Outcome and risk fac tors of hepatic artery thrombosis after orthotopic liver transplantation in adults. Transplant Proc, 2001; 33: 2712-13

3. Song S, Kwon CHD, Moon HH et al: Single-center experience of consecutive 522 cases of hepatic artery anastomosis in living donor liver transplantation. Transplant Proc, 2015; 47: 1905-11

4. Ahn CS, Hwang S, Moon DB et al: Right gastroepiploic artery is the first alternative inflow source for hepatic arterial reconstruction in living donor liver transplantation. Transplant Proc, 2012; 44: 451-53

5. Ahn CS, Lee SG, Hwang S et al: Anatomic variation of the right hepatic artery and its reconstruction for living donor liver transplantation using right lobe graft. Transplant Proc, 2005; 37: 1067-69

6. Miyagi S, Enomoto Y, Sekiguchi S et al: Microsurgical back wall support suture technique with double needle sutures on hepatic artery reconstruction in living donor liver transplantation. Transplant Proc, 2008; 40: 2521-22

7. Bekker J, Ploem S, de Jong KP: Early hepatic artery thrombosis after liver transplantation: A systematic review of the incidence, outcome and risk factors. Am J Transplant, 2009; 9: 746-57
8. Vrochides D, Hassanain M, Metrakos $P$ et al: Re-vascularization may not increase graft survival after hepatic artery thrombosis in liver transplant recipients. Hippokratia, 2010; 14: 115-18

9. Uchiyama H, Shirabe K, Taketomi A et al: Extra-anatomical hepatic artery reconstruction in living donor liver transplantation: Can this procedure save hepatic grafts? Liver Transpl, 2010; 16: 1054-61

10. Ikegami T, Kawasaki S, Hashikura Y et al: An alternative method of arterial reconstruction after hepatic arterial thrombosis following living-related liver transplantation. Transplantation, 2000; 69: 1953-55

11. Katz E, Fukuzawa K, Schwartz $M$ et al: The splenic artery as the inflow in arterial revascularization of the liver graft in clinical liver transplantation. Transplantation, 1992; 53: 1373-74

12. Asakura T, Ohkohchi $\mathrm{N}$, Orii T et al: Arterial reconstruction using vein graft from the common iliac artery after hepatic artery thrombosis in living-related liver transplantation. Transplant Proc, 2000; 32: 2250-51

13. Wang CC, Lin TS, Chen $\mathrm{CL}$ et al: Arterial reconstruction in hepatic artery occlusions in adult living donor liver transplantation using gastric vessels. Surgery, 2008; 143: 686-90

14. Lee JH, Oh DY, Seo JW et al: Versatility of right gastroepiploic and gastroduodenal arteries for arterial reconstruction in adult living donor liver transplantation. Transplant Proc, 2011; 43: 1716-19 